\title{
IL-6 Protein Expression is Increased in Fast Glycolytic Muscle Fibers Over Time in the SOD1G93A Mouse model.
}

Laura Moreno Martínez ( $\sim$ lauramm@unizar.es)

Universidad de Zaragoza https://orcid.org/0000-0002-7277-4318

\section{Ana Cristina Calvo}

Universidad de Zaragoza Facultad de Veterinaria https://orcid.org/0000-0001-5193-7782

\section{Leticia Moreno-García}

Universidad de Zaragoza Facultad de Veterinaria

\section{Gabriel García-Salamero}

12th of October Hospital Research Institute: Instituto de Investigacion Hospital 12 de Octubre

\section{Christian Lunetta}

NEMO Clinical Center

\section{Silvana Penco}

Niguarda Ca Granda Hospital

\section{Claudia Tarlarini}

Niguarda Ca Granda Hospital

\section{Pilar Zaragoza}

Universidad de Zaragoza Facultad de Veterinaria

\section{Alberto García-Redondo}

12th of October Hospital Research Institute: Instituto de Investigacion Hospital 12 de Octubre

\section{Rosario Osta}

Universidad de Zaragoza Facultad de Veterinaria

\section{Research Article}

Keywords: Amyotrophic lateral sclerosis, interleukin 6, SOD1G93A, spinal cord, skeletal muscle, neurodegenerative disorders.

Posted Date: August 9th, 2021

DOI: https://doi.org/10.21203/rs.3.rs-785505/v1

License: (c) (1) This work is licensed under a Creative Commons Attribution 4.0 International License. Read Full License 


\section{Abstract}

The deregulation of the inflammatory cytokine interleukin (IL)- 6 has been associated to a variety of neurodegenerative diseases, including amyotrophic lateral sclerosis (ALS). The aim of this work was to analyze the variation of IL-6 levels in blood and damaged tissues during the course of the disease. We studied IL-6 protein expression in spinal cord, extensor digital longus (EDL) muscle and soleus (SOL) muscle of the SOD1G93A animal model at four stages of the disease by western blot. Concurrently, we analyzed IL- 6 gene and protein expression in blood of ALS patients, healthy subjects and patients with other neuropathies through RTqPCR and ELISA. The results revealed different expression patterns depending on both the tissue analyzed and the stage of the disease, showing increasing IL- 6 levels in EDL muscle over time. Moreover, lower IL- 6 levels in blood were found in ALS patients. The decreased levels of IL- 6 in blood from ALS patients could suggest that IL- 6 might not be the main pro-inflammatory biomarker in the last stages in whole blood. In contrast, IL- 6 may play a main role in fast glycolytic muscle fibers associated with muscle atrophy, suggesting that modulation of IL-6 in this tissue could be a potential target for anti-inflammatory therapies in ALS.

\section{Introduction}

Amyotrophic lateral sclerosis (ALS) is a fatal neurodegenerative disease characterized by a progressive motor neuron degeneration leading to muscle paralysis and death within 2-5 years after diagnosis. Although few patients have familial disease, the cause of the majority of ALS cases is unknown [1]. To date, there are two treatments approved for ALS, riluzole and edaravone, although they only extend life expectancy for few months [2]. ALS is considered a heterogeneous disease with multiple pathological mechanisms involved, such as impaired protein homeostasis, aberrant RNA metabolism, excitotoxicity, mitochondrial dysfunction or neuroinflammation [3].

Inflammation in ALS has been widely studied during the last two decades evidencing its key role in the progression of ALS. A complex interplay between immune cells and their mediators seems to promote either disease progression or neuroprotection [4]. A better understanding of this challenging scenario could shed light on the inflammatory mechanisms which exacerbate the disease course and would help in the identification of new biomarkers and therapeutic targets. In this sense, numerous circulating cytokines have been found deregulated in ALS [5], suggesting they could be mediating diverse inflammatory pathways. One of the cytokines most frequently found altered is interleukin (IL)-6. IL-6 has pleiotropic functions and can exert both pro-inflammatory and anti-inflammatory actions in different tissues and organs [6]. Regarding ALS, in the SOD1G93A mouse model we found that increased IL-6 levels in plasma measured at the symptomatic and terminal stages were correlated with the longevity of mice, suggesting that higher levels of IL- 6 could be associated with faster progression of the disease [7]. In addition, multiple studies have assessed IL- 6 protein levels in blood from ALS patients and controls, obtaining very variable results. Unlike blood, few studies addressed the cytokine profile in tissues, leaving an open field to explore. 
Given the little knowledge about the cytokine status in tissues different from blood, in this work we analyzed the protein expression of IL-6 in spinal cord, extensor digitorum longus (EDL) muscle and soleus (SOL) muscle at four stages of the disease in the SOD1G93A mouse model and their wildtype (WT) controls. Moreover, we studied IL-6 expression at protein and gene level in blood from a cohort of ALS patients, patients with other neuropathies (ONP) and healthy subjects to shed light on the support for diagnosis role of IL-6 at clinical level.

\section{Materials And Methods}

\section{Animals and sampling}

Transgenic mice were obtained by mating hemizygous B6SJL-Tg SOD1G93A males (stock number 002726) purchased from The Jackson Laboratory (Bar Harbor, ME, USA) with C57BL/6J x SJL/J F1 hybrid females (B6SJLF1) purchased from Janvier Labs (Saint- Berthevin Cedex, France). The offspring were identified by PCR assay as described in The Jackson Laboratory protocol. The mice were housed at the animal facilities in Centro de Investigación Biomédica de Aragón (CIBA) under a standard light:dark (12:12) cycle. Food and water were provided ad libitum.

Spinal cord, EDL muscle and SOL muscle were isolated from a total of thirty-two WT and SOD1G93A mice and their controls after euthanized with $\mathrm{CO}_{2}$ at four stages of the disease: pre-symptomatic (P40), early symptomatic (P60), symptomatic (P90) and late symptomatic (P120). These tissues were immediately frozen in dry ice and stored at $-80 \circ \mathrm{C}$ until its processing.

All procedures were approved by the Ethic Committee for Animal Experiments from the University of Zaragoza. The care and use of animals were performed accordingly with the Spanish Policy for Animal Protection RD53/2013, which meets the European Union Directive 2010/63 on the protection of animals used for experimental and other scientific purposes.

\section{Experimental design involving patients}

This study included a total of sixty participants divided in three groups: twenty ALS patients, twenty healthy controls and twenty ONP patients originating from the Clinical Center NEMO, Milan, Italy. The clinical characteristics of the participants in each group were firstly assessed. In the ALS group, these characteristics included the revised ALS Functional Rating Scale-revised (ALSFRS-r), the age at onset, age at sampling, gender, the onset site and the genetic diagnosis (Table 1). The ALSFRS-r provided an estimation of the patient's degree of functional impairment. 
Table 1

General and clinical characteristics of the subjects involved in the study. All ALS patients showed 48 of ALSFRS-r at the moment of taking the sample and present mutations in the following genes: Sod1, Fus, Tardbp and C9orf72

\begin{tabular}{|llll|}
\hline Patients' characteristics & $\begin{array}{l}\text { ALS patients } \\
(\mathbf{n = 2 0})\end{array}$ & $\begin{array}{l}\text { ONP patients } \\
(\mathbf{n}=\mathbf{2 0})\end{array}$ & $\begin{array}{l}\text { Control subjects } \\
(\mathbf{n}=\mathbf{2 0})\end{array}$ \\
\hline Gender (n) & $\begin{array}{l}13 \text { males } \\
7 \text { females }\end{array}$ & $\begin{array}{l}12 \text { males } \\
8 \text { females }\end{array}$ & $\begin{array}{l}7 \text { males } \\
13 \text { females }\end{array}$ \\
\hline Age at illness onset (mean \pm SD) & $64 \pm 8.43$ & & \\
\hline Disease duration, months (mean \pm SD) & $26 \pm 17.56$ & & \\
\hline Age at sampling (mean \pm SD) & $66 \pm 8.67$ & $56 \pm 12.08$ & \\
\hline Site at illness onset (n patients) & & & \\
\hline Bulbar & 4 & & \\
\hline Upper limb & 5 & & \\
\hline Lower limb & 8 & & \\
\hline Generalized & 2 & & \\
\hline Respiratory & 1 & & \\
\hline ALS = amyotrophic lateral sclerosis, ONP $=$ other neuropathies, SD = standard deviation.
\end{tabular}

Whole blood samples from ALS patients, ONP patients and control subjects were received frozen in PAXgene ${ }^{\mathrm{Tm}}$ tubes (BD Biosciences, USA), which were stored at $-80^{\circ} \mathrm{C}$ until used. Blood samples from patients were obtained with written informed consent prior to inclusion in the study, which has been conducted according to Declaration of Helsinki principles, and following the Directive 2004/23/EC of the European Parliament and of the Council. Participants were identified by number, not by name. Procedures with the samples were approved by the Ethics Committee for Clinical Research of Aragon (CEICA) (PI18/078).

\section{Western blotting}

Spinal cord, EDL and SOL from eight WT and SOD1G93A mice, per stage, were ground into a powder using the Tissue Lyser LT (Qiagen). Then, powdered tissues were resuspended in RIPA lysis buffer together with protease inhibitors (SC-24948, Santa Cruz Biotechnology, Inc., CA, USA) according to manufacturer's protocol. Total protein was quantified using the BCA method (Sigma-Aldrich). A total of $30 \mathrm{\mu g}$ of protein was loaded into a 10\% SDS-PAGE gel to analyze the protein expression of IL- 6 . After electrophoresis, proteins were transferred to a PVDF membrane (Amersham ${ }^{\mathrm{TM}}$, GE Healthcare Life Sciences) and subsequently blocked with a Tris-buffered saline solution containing $5 \%$ bovine serum albumin (BSA) and $0.1 \%$ Tween as supplement for $1 \mathrm{~h}$ at room temperature. Then, membranes were 
incubated overnight at $4^{\circ} \mathrm{C}$ with the primary antibody for IL-6 (PK-AB815-61632M, Promokine). The housekeeping proteins selected to normalize the results were actin beta actin (ACTB) (PA1-183, Thermo Fisher Scientific) for spinal cord samples, and glyceraldehyde-3-phosphate dehydrogenase (GAPDH) (PKAB718-3781, Promokine) for muscle samples. Secondary antibody used was goat anti-Rabbit IgG (31466, Thermo Fisher Scientific). Finally, chemiluminescence detection was performed using Immobilon Crescendo Western HRP Substrate (Millipore) in a Molecular Imager ${ }^{\circledR}$ VersaDoc ${ }^{\text {TM MP }} 4000$ system. The computer-assisted analysis of the bands was performed with AlphaEase FC software (Bonsai Technologies Group, S.A., Madrid, Spain).

\section{RTqPCR}

The PAXgene Blood RNA Kit (PreAnalytiX, 8634 Hombrechtikon, Switzerland) was used to extract the RNA fraction from blood samples from ALS patients $(n=18)$, ONP patients $(n=20)$ and healthy subjects $(n=$ 20), following the manufacturer's instructions. After obtaining purified intracellular RNA, cDNA was synthesized using the kit qScript ${ }^{\text {TM }}$ cDNA SuperMix (Quanta BioScience, Inc.). Following this, gene expression of IL- 6 was quantified through qPCR using the TaqMan® probe for IL-6 (Hs00985639_m1, Applied Biosystems Inc.) mixed with TaqMan ${ }^{\circledR}$ Fast Universal PCR Master Mix (Applied Biosystems Inc.). Three endogenous genes, glyceraldehyde 3-phosphate dehydrogenase (GAPDH) (Hs99999905_m1), hypoxanthine phosphoribosyltransferase (HPRT) (Hs02800695_m1), and TATA-binding protein, (TBP) (Hs00427620_m1) were used to normalize the target gene expression. The $\Delta \Delta C T$ method was used to determine relative changes in transcriptional expression [8].

\section{ELISA}

The same samples used in the qPCR analysis were analyzed to perform ELISA assays. Whole blood in PAXgene ${ }^{\text {TM }}$ tubes from patients was centrifuged at the initial step and the plasma phase was collected in new tubes. In this plasma phase, IL-6 protein expression was measured through an ELISA (BMS213-2, Invitrogen, Thermo Fisher Scientific Inc) in those plasma samples from ALS patients $(n=14)$, ONP patients $(n=20)$ and healthy subjects $(n=18)$. The protocol was performed following the manufacturer's instructions.

\section{Statistical analysis}

Data distribution was analyzed by Shapiro-Wilk test. Accordingly, results obtained in the western blot analysis were compared using t-tests or Mann-Whitney U-tests depending on data distribution. RTqPCR results were analyzed using one-way ANOVA test and Bonferroni post-hoc. Chi-squared test was used to analyze the results obtained in the ELISA used to measure IL-6 in plasma samples from ALS patients. Statistical analysis was performed using SPSS (version 20, IBM, Armonk, NY). Graphs were elaborated using GraphPad Prism 157 Software (version 5, La Jolla, CA). All of the values were expressed as the mean \pm standard error of the mean (SEM) or standard deviation (SD). Significance levels were set at $p<$ 0.05 . 


\section{Results}

\section{Expression of IL-6 varies along disease and tissue in SOD1G93A mice}

Protein expression of IL- 6 was studied in two of the most affected tissues in the disease: spinal cord and skeletal muscle. The western blot revealed different patterns of expression depending on both the stage of the disease and the tissue studied (Fig. 1). Regarding the spinal cord, lower levels of IL-6 were found in SOD1G93A mice at the pre-symptomatic (P40) and late symptomatic (P120) stages compared to the levels obtained in WT mice. On the other hand, two different skeletal muscles were studied: the fasttwitch EDL muscle and the slow-twitch SOL muscle. In EDL we could observe that IL-6 expression was increased in the SOD1G93A mice from the early symptomatic stage (P60) to the terminal stage. In contrast, we found a little tendency $(p<0.067)$ for IL-6 to be increased in SOL from SOD1G93A mice at P40, although the levels were similar in WT and SOD1G93A mice at the other stages of the disease.

\section{IL-6 gene expression was downregulated in whole blood from ALS patients}

IL- 6 was measured in the RNA fraction from whole blood samples of ALS patients, ONP patients and control subjects by qPCR. The analysis showed a significant decreased of IL-6 levels in the ALS patients group compared to levels found in healthy subjects group. However, no significant differences were found between ONP and ALS patients (Fig. 2).

\section{Absence of IL-6 protein levels in plasma was associated with ALS}

IL-6 protein levels were analyzed in the plasma phase from ALS patients, ONP patients and healthy subjects. After performing the ELISAs, we observed in several samples that IL-6 levels were under the limit of detection of the assay. Given that IL-6 protein concentration was well quantified in half of the samples, we considered that there was very little or null IL-6 protein in those samples that the expression was not detected. Thus, we analyzed the association of the presence (detection) or absence (no detection) of IL-6 with ALS patients, ONP patients and control group. The statistical analysis using Chi-squared test revealed a significant relation between the absence of IL- 6 in blood and the ALS patients group, $\chi^{2}(2, N=$ $52)=7.241, p=0.027$. The percentage of ALS patients where IL- 6 was not detected was $90 \%(13 / 14)$, whereas the percentages found for ONP and control patients were $60 \%(11 / 20)$ and $50 \%(9 / 18)$, respectively (Fig. 3).

\section{Discussion}

The cytokine IL-6 has been widely studied in a wide range of neurodegenerative disorders, including ALS. However, there is still the need to elucidate the exact role of IL- 6 during the disease in different tissues. In this work we have analyzed the IL-6 protein expression in two of the most damaged tissues in the 
SOD1G93A mouse model, the spinal cord and the skeletal muscle, at different stages of the disease to assess its evolution over time. In addition, we have measured IL- 6 gene and protein expression levels in blood from ALS patients to study whether or not IL- 6 could be considered a marker of the peripheral inflammatory response in ALS.

Our results showed lower IL-6 protein levels in spinal cord at the pre-symptomatic and terminal stages in SOD1G93A mice. On the one hand, this finding supports the early anti-inflammatory or neuroprotective compensatory response found in central nervous system (CNS) [9]. On the other hand, the decreased expression found at the final stage is not in accordance with a unique previous study reporting increased IL-6 levels in the spinal cord of SOD1G93A mice [10]. An explanation for the lower levels of IL-6 found may be that the progressive change to a pro-inflammatory status occurring in later stages of ALS could be governed through other pro-inflammatory cytokines, such as IL-1 $\beta$ and tumor necrosis factor (TNF)- $a$ [11], having IL-6 a less relevant effect. On the other hand, the predominant inflammatory status in the terminal stage in ALS could be explained by higher IL-6 mRNA expression, which could not be translated to protein due to the aberrant RNA processing occurring in ALS, as previously observed in the SOD1G93A mouse model [12]. Nevertheless, very little has been found in the literature about the evolution of this cytokine in the spinal cord in ALS over time and further research could help to better understand the role of this mediator in the spinal cord.

Regarding the skeletal muscle, we found increasing levels of IL-6 from the symptomatic stage to the terminal stage in EDL, but not in SOL. Unlike SOL, EDL is mostly composed of fast-twitch and glycolytic fibers. Previous studies have shown that IL- 6 is a myokine whose expression is affected by fiber type [13, 14]. For instance, under no disease condition, IL- 6 release was only inducible from SOL but not EDL muscle, leading to higher IL-6 protein levels in slow oxidative muscle fibers [13]. However, fast glycolytic fibers (EDL) are more vulnerable than slow-twitch oxidative fibers (SOL) under a variety of atrophic conditions [15], such as those occurring in ALS. In addition, in ALS, activated inflammation and abnormal glial responses occur in the limb muscle [16]. Therefore, our finding showing an unusual increase of IL-6 in EDL could indicate that the inherent pathological processes of ALS are responsible for this stimulation. In addition, IL-6 can perform as an anti-inflammatory myokine [17], suggesting that increased IL-6 release could represent a neuroprotective reaction against the excitotoxicity damage [18], as observed in an ALS animal model [19]. In this sense, the progressive muscle atrophy may be accompanied by increasing IL-6 levels in EDL reflecting the damage suffered by this type of muscles. Interestingly, inactivation of STAT3IL6 signaling in fibro-adipogenic progenitors, activated in response to muscle injury, effectively countered muscle atrophy and fibrosis in mouse models of acute denervation and SODG93A mice [20]; this suggests that IL- 6 could be a potential target to be considered in ALS therapies. Accordingly, previous studies have shown that neuroprotective molecules such as the fragment $C$ of tetanus toxin may directly act on modulation of IL-6, improving the disease condition in the SOD1G93A mouse model [21].

In relation to circulating IL-6, we analyzed both gene and protein expression of IL-6 in blood samples from patients. We found significant lower levels of IL-6 mRNA in ALS patients compared to the levels obtained in healthy subjects. This result is in agreement with a previous study where they found a downregulation 
of 116 gene expression in the peripheral blood of sporadic ALS patients [22]. Similarly, at protein level we found that the absence of IL- 6 in blood was significantly associated with the ALS patients group. Despite this association, the no detection of IL- 6 could be caused by the very low concentration of this protein found in blood due to the method used to collect the blood samples, PAX tubes, which could be interfering in the integrity of this cytokine. Therefore, the method employed to extract protein from the whole blood may imply a limitation on this study. Although PAX tubes are proper and reliable for mRNA analysis, other methods should be considered for protein study. In addition, the few mRNA transcripts found in blood of ALS patients could be missed in the translation to protein due to a dysfunction in the RNA processing, which is similar to our findings related to the spinal cord of the mouse model. A great variability of protein levels of this cytokine in blood has been found in the literature. Although many studies reported that higher levels were associated with ALS and correlated with faster progression of the disease course $[5,19,23-26]$, others found no significant differences $[4,27,28]$ or even decreasing IL-6 levels in ALS patients over time [29]. These controversial results found in blood suggest that this inflammatory mediator could be susceptible to variations due to processes not dependent on ALS, e.g. the gender and obesity [30]. In addition, a recent study reported blood IL-6 protein levels increased with aging in both ALS patients and healthy controls [4]. In the same study, they also found an association of higher IL-6 levels with respiratory dysfunction, proposing that this cytokine could be explored as a marker of respiratory failure [4]. In view of our results, another point to consider when analyzing IL-6 protein levels in blood would be the methodology followed from obtaining the whole blood from patients to the ELISA, as it may have a great influence on the results. Therefore, the instability of circulating IL- 6 could become a barrier for its consideration by itself as a specific biomarker of ALS.

In our study, the most relevant finding is a consistent increasing protein IL-6 level in EDL in the SOD1G93A mouse model over time, which could correlate with the atrophy progression, and could be an indicator of an anti-inflammatory response to damage by exacerbating the inflammatory status in the muscle. In contrast, we found lower protein IL-6 expression in the spinal cord at pre-symptomatic and terminal stages as well as lower mRNA IL- 6 expression in blood of ALS patients, suggesting that IL- 6 might not be the main pro-inflammatory mediator in these tissues. On the other hand, given the controversial results found in blood in the literature, we suggest that tissues damaged directly by the disease, such as the skeletal muscle predominantly composed of fast glycolytic muscle fibers, could provide a closer insight of the mechanisms involved in ALS. Accordingly, future studies focused on the study of the role of IL-6 in the skeletal muscle of ALS patients by muscle biopsies could be significance. Although further research is needed to shed light on this, IL- 6 could be a potential indicator of damage and the imbalanced inflammatory status of fast glycolytic muscle fibers in ALS.

\section{Declarations}

Funding: This research was funded by Instituto de Salud Carlos III, PI17/00949, and Fondo Europeo de Desarrollo Regional (FEDER) "Una manera de hacer Europa” from the European Union, Centro de Investigación Biomédica en Red sobre Enfermedades Neurodegenerativas (CIBERNED, CB18/05/0037), Fundación "Federación Española de Enfermedades Raras" (FEDER), and Consoli-dated Groups from 
Gobierno de Aragón. LM-M was supported by Departamento de Industria e Innovación from Gobierno de Aragón and Fondo Social Europeo.

Conflicts of interest/Competing interests: The authors declare that they have no conflict of interest.

Availability of data and material: The datasets used and analyzed during the current study are available from the corresponding author on reasonable request.

Code availability: Not applicable.

Authors' contributions: Conceptualization, A.G.-R. and R.O.; methodology, C.L., S.P., C.T., G.G.-S., L.M.-G. and L.M.-M..; software, A.C.C. and L.M.-M.; validation, A.C.C., A.G.-R. and R.O.; formal analysis, A.C.C. and L.M.-M.; investigation, A.C.C. and L.M.-M.; resources, A.C.C, A.G.-R., C.L., S.P., C.T. and R.O; data curation, A.C.C., L.M.-M. and R.O.; writing-original draft preparation, L.M.-M.; writing-review and editing, A.C.C and R.O.; visualization, C.L., S.P., C.T., G.G.-S., L.M.-G. and P.Z.; supervision, A.G.-R. and R.O.; project administration, A.G.-R. and R.O.; funding acquisition, A.C.C, A.G.-R., P.Z. and R.O. All authors have read and agreed to the published version of the manuscript.

Ethics approval: All procedures with animals were approved by the Ethic Committee for Animal Experiments from the University of Zaragoza. The care and use of animals were performed accordingly with the Spanish Policy for Animal Protection RD53/2013, which meets the European Union Directive 2010/63 on the protection of animals used for experimental and other scientific purposes. Samples from patients were obtained with written informed consent prior to inclusion in the study, which has been conducted according to Declaration of Helsinki principles, and following the Directive 2004/23/EC of the European Parliament and of the Council. Participants were identified by number, not by name. Procedures with the samples were approved by the Ethics Committee for Clinical Research of Aragon (CEICA) (PI18/078).

Consent to participate: All participants gave their informed consent prior to their inclusion in the study.

Consent for publication: All participants signed the informed consent regarding publishing their data.

\section{References}

1. Hardiman O, Al-Chalabi A, Chio A, Corr EM, Logroscino G, Robberecht W, Shaw PJ, Simmons Z, van den Berg LH (2017) Amyotrophic lateral sclerosis. Nat Rev Dis Primers 3:17071. https://doi.org/10.1038/nrdp.2017.71

2. Jaiswal MK (2019) Riluzole and edaravone: A tale of two amyotrophic lateral sclerosis drugs. Med Res Rev 39:733-748. https://doi.org/10.1002/med.21528

3. Turner MR, Hardiman O, Benatar M, Brooks BR, Chio A, de Carvalho M, Ince PG, Lin C, Miller RG, Mitsumoto H, Nicholson G, Ravits J, Shaw PJ, Swash M, Talbot K, Traynor BJ, Van den Berg LH, 
Veldink JH, Vucic S, Kiernan MC (2013) Controversies and priorities in amyotrophic lateral sclerosis. Lancet Neurol 12:310-322. https://doi.org/10.1016/S1474-4422(13)70036-X

4. Pronto-Laborinho A, Pinto S, Gromicho M, Pereira M, Swash M, de Carvalho M (2019) Interleukin-6 and amyotrophic lateral sclerosis. J Neurol Sci 398:50-53.

https://doi.org/10.1016/j.jns.2019.01.026

5. Hu Y, Cao C, Qin XY, Yu Y, Yuan J, Zhao Y, Cheng Y (2017) Increased peripheral blood inflammatory cytokine levels in amyotrophic lateral sclerosis: a meta-analysis study. Sci Rep 7:9094-017-09097-1 https://doi.org/10.1038/s41598-017-09097-1

6. Scheller J, Chalaris A, Schmidt-Arras D, Rose-John S (2011) The pro- and anti-inflammatory properties of the cytokine interleukin-6. Biochim Biophys Acta 1813:878-888.

https://doi.org/10.1016/j.bbamcr.2011.01.034

7. Moreno-Martinez L, de la Torre M, Toivonen JM, Zaragoza P, Garcia-Redondo A, Calvo AC, Osta R (2019) Circulating Cytokines Could Not Be Good Prognostic Biomarkers in a Mouse Model of Amyotrophic Lateral Sclerosis. Front Immunol 10:801. https://doi.org/10.3389/fimmu.2019.00801

8. Livak KJ, Schmittgen TD (2001) Analysis of relative gene expression data using real-time quantitative PCR and the 2(-Delta Delta C(T)) Method. Methods 25:402-408.

https://doi.org/10.1006/meth.2001.1262

9. Hooten KG, Beers DR, Zhao W, Appel SH (2015) Protective and Toxic Neuroinflammation in Amyotrophic Lateral Sclerosis. Neurotherapeutics 12:364-375. https://doi.org/10.1007/s13311-0140329-3

10. Hensley K, Fedynyshyn J, Ferrell S, Floyd RA, Gordon B, Grammas P, Hamdheydari L, Mhatre M, Mou S, Pye QN, Stewart C, West M, West S, Williamson KS (2003) Message and protein-level elevation of tumor necrosis factor alpha (TNF alpha) and TNF alpha-modulating cytokines in spinal cords of the G93A-SOD1 mouse model for amyotrophic lateral sclerosis. Neurobiol Dis 14:74-80. https://doi.org/10.1016/s0969-9961(03)00087-1

11. Jeyachandran A, Mertens B, McKissick EA, Mitchell CS (2015) Type I Vs. Type II Cytokine Levels as a Function of SOD1 G93A Mouse Amyotrophic Lateral Sclerosis Disease Progression. Front Cell Neurosci 9:462. https://doi.org/10.3389/fncel.2015.00462

12. Manzano R, Toivonen JM, Calvo AC, Olivan S, Zaragoza P, Rodellar C, Montarras D, Osta R (2013) Altered in vitro proliferation of mouse SOD1-G93A skeletal muscle satellite cells. Neurodegener Dis 11:153-164. https://doi.org/10.1159/000338061

13. Liang AP, Drazick AT, Gao H, Li Y (2018) Skeletal muscle secretion of IL-6 is muscle type specific: Ex vivo evidence. Biochem Biophys Res Commun 505:146-150.

https://doi.org/10.1016/j.bbrc.2018.09.042

14. Jia WH, Wang NQ, Yin L, Chen X, Hou BY, Qiang GF, Chan CB, Yang XY, Du GH (2019) Effect of skeletal muscle phenotype and gender on fasting-induced myokine expression in mice. Biochem Biophys Res Commun 514:407-414. https://doi.org/10.1016/j.bbrc.2019.04.155 
15. Wang Y, Pessin JE (2013) Mechanisms for fiber-type specificity of skeletal muscle atrophy. Curr Opin Clin Nutr Metab Care 16:243-250. https://doi.org/10.1097/MCO.0b013e328360272d

16. Van Dyke JM, Smit-Oistad IM, Macrander C, Krakora D, Meyer MG, Suzuki M (2016) Macrophagemediated inflammation and glial response in the skeletal muscle of a rat model of familial amyotrophic lateral sclerosis (ALS). Exp Neurol 277:275-282. https://doi.org/10.1016/j.expneurol.2016.01.008

17. Pedersen BK, Febbraio MA (2008) Muscle as an endocrine organ: focus on muscle-derived interleukin-6. Physiol Rev 88:1379-1406. https://doi.org/10.1152/physrev.90100.2007

18. Ali C, Nicole O, Docagne F, Lesne S, MacKenzie ET, Nouvelot A, Buisson A, Vivien D (2000) Ischemiainduced interleukin- 6 as a potential endogenous neuroprotective cytokine against NMDA receptormediated excitotoxicity in the brain. J Cereb Blood Flow Metab 20:956-966. https://doi.org/10.1097/00004647-200006000-00008

19. Lu CH, Allen K, Oei F, Leoni E, Kuhle J, Tree T, Fratta P, Sharma N, Sidle K, Howard R, Orrell R, Fish M, Greensmith L, Pearce N, Gallo V, Malaspina A (2016) Systemic inflammatory response and neuromuscular involvement in amyotrophic lateral sclerosis. Neurol Neuroimmunol Neuroinflamm 3:e244. https://doi.org/10.1212/NXI.0000000000000244

20. Madaro L, Passafaro M, Sala D, Etxaniz U, Lugarini F, Proietti D, Alfonsi MV, Nicoletti C, Gatto S, De Bardi M, Rojas-Garcia R, Giordani L, Marinelli S, Pagliarini V, Sette C, Sacco A, Puri PL (2018) Denervation-activated STAT3-IL-6 signalling in fibro-adipogenic progenitors promotes myofibres atrophy and fibrosis. Nat Cell Biol 20:917-927. https://doi.org/10.1038/s41556-018-0151-y

21. Moreno-Martinez L, de la Torre M, Muñoz MJ, Zaragoza P, Aguilera J, Calvo AC, Osta R (2020) Neuroprotective Fragment C of Tetanus Toxin Modulates IL- 6 in an ALS Mouse Model. Toxins (Basel) 12:330. doi: 10.3390/toxins12050330 https://doi.org/10.3390/toxins 12050330

22. Andres-Benito P, Moreno J, Dominguez R, Aso E, Povedano M, Ferrer I (2017) Inflammatory Gene Expression in Whole Peripheral Blood at Early Stages of Sporadic Amyotrophic Lateral Sclerosis. Front Neurol 8:546. https://doi.org/10.3389/fneur.2017.00546

23. Ngo ST, Steyn FJ, Huang L, Mantovani S, Pfluger CM, Woodruff TM, O'Sullivan JD, Henderson RD, McCombe PA (2015) Altered expression of metabolic proteins and adipokines in patients with amyotrophic lateral sclerosis. J Neurol Sci 357:22-27. https://doi.org/10.1016/j.jns.2015.06.053

24. Blasco H, Garcon G, Patin F, Veyrat-Durebex C, Boyer J, Devos D, Vourc'h P, Andres CR, Corcia P (2017) Panel of Oxidative Stress and Inflammatory Biomarkers in ALS: A Pilot Study. Can J Neurol Sci 44:90-95. https://doi.org/10.1017/cjn.2016.284

25. Guo J, Yang X, Gao L, Zang D (2017) Evaluating the levels of CSF and serum factors in ALS. Brain Behav 7:e00637. https://doi.org/10.1002/brb3.637

26. Prado LGR, Rocha NP, de Souza LC, Bicalho ICS, Gomez RS, Vidigal-Lopes M, Braz NFT, Vieira ELM, Teixeira AL (2018) Longitudinal assessment of clinical and inflammatory markers in patients with amyotrophic lateral sclerosis. J Neurol Sci 394:69-74. https://doi.org/10.1016/j.jns.2018.08.033 
27. Vlam L, Stam M, de Jager W, Cats EA, van den Berg LH, van der Pol WL (2015) Cytokine profiles in multifocal motor neuropathy and progressive muscular atrophy. J Neuroimmunol 286:1-4. https://doi.org/10.1016/j.jneuroim.2015.06.008

28. Martinez-Merino L, Iridoy M, Galbete A, Roldan M, Rivero A, Acha B, Irun P, Canosa C, Pocovi M, Mendioroz M, Jerico I (2018) Evaluation of Chitotriosidase and CC-Chemokine Ligand 18 as Biomarkers of Microglia Activation in Amyotrophic Lateral Sclerosis. Neurodegener Dis 18:208-215. https://doi.org/10.1159/000490920

29. Ehrhart J, Smith AJ, Kuzmin-Nichols N, Zesiewicz TA, Jahan I, Shytle RD, Kim SH, Sanberg CD, Vu TH, Gooch CL, Sanberg PR, Garbuzova-Davis S (2015) Humoral factors in ALS patients during disease progression. J Neuroinflammation 12:127-015-0350-4 https://doi.org/10.1186/s12974-015-0350-4

30. Martinez-Garcia MA, Moncayo S, Insenser M, Montes-Nieto R, Fernandez-Duran E, Alvarez-Blasco F, Luque-Ramirez M, Escobar-Morreale HF (2019) Postprandial inflammatory responses after oral glucose, lipid and protein challenges: Influence of obesity, sex and polycystic ovary syndrome. Clin Nutr. https://doi.org/10.1016/j.clnu.2019.03.027

\section{Figures}


a

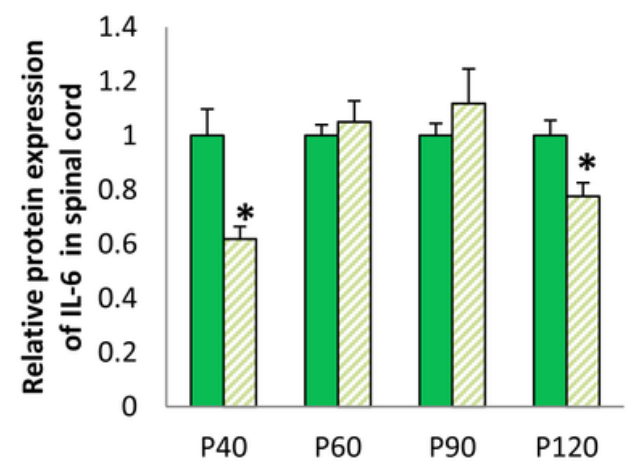

c

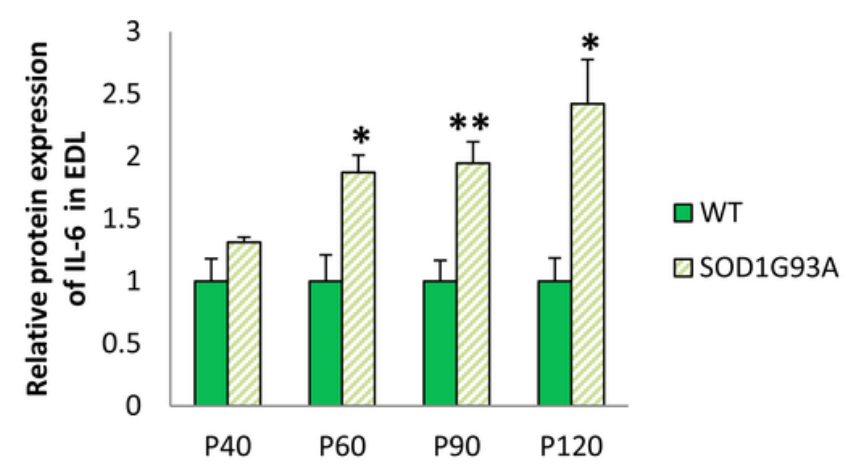

e
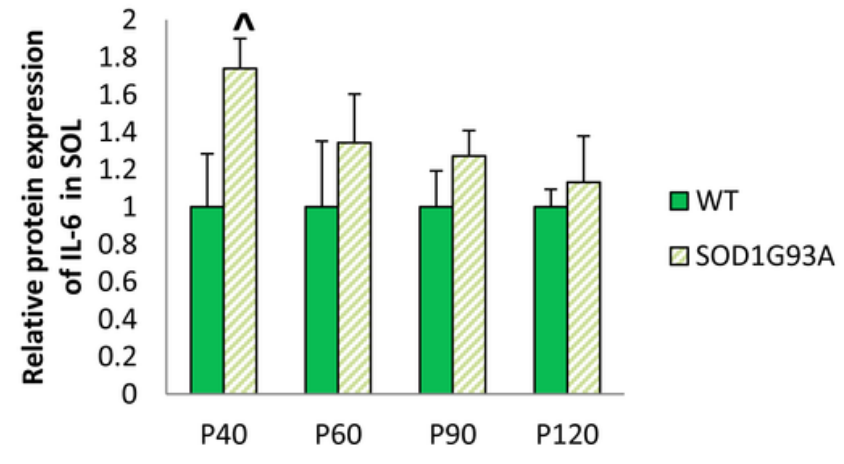

b

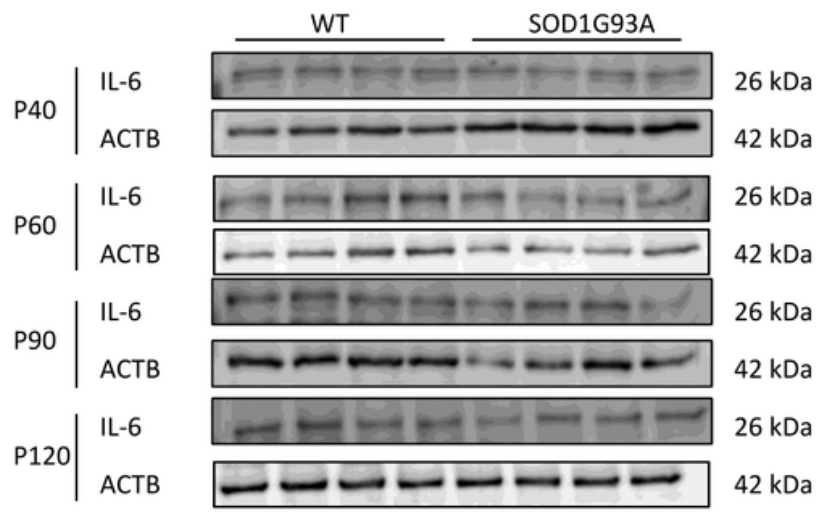

d

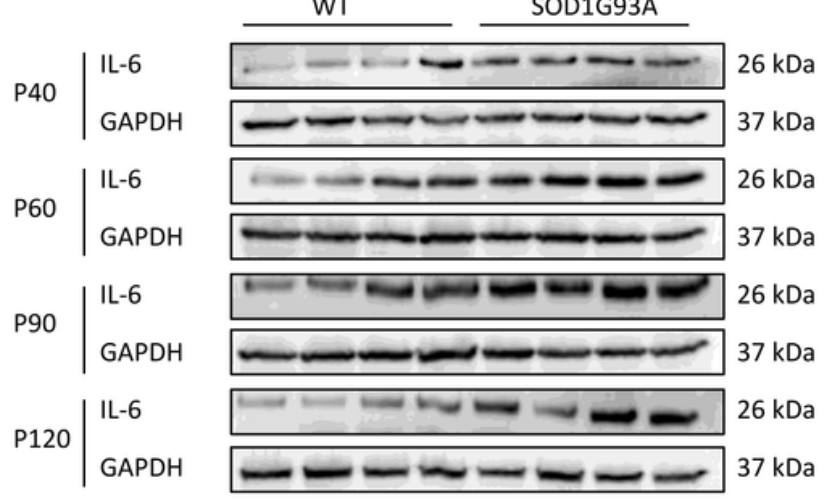

f f \begin{tabular}{l|l} 
P40 & IL-6 \\
GAPDH
\end{tabular}

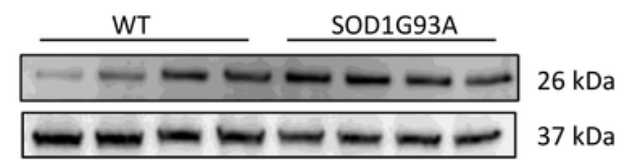
\begin{tabular}{l|l} 
P60 & IL-6 \\
GAPDH
\end{tabular} \begin{tabular}{l|l} 
P90 & $\begin{array}{l}\text { IL-6 } \\
\text { GAPDH }\end{array}$ \\
P120 & $\begin{array}{l}\text { IL-6 } \\
\text { GAPDH }\end{array}$
\end{tabular}

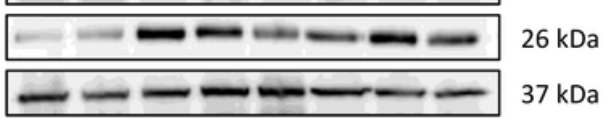

$=-20=00 \mathrm{mba}$ 37 kDa $-\infty-m-\infty \mathrm{kDa}$ $37 \mathrm{kDa}$

\section{Figure 1}

Relative protein expression of IL-6 in spinal cord, EDL and SOL from WT and SOD1G93A transgenic mice at four stages of the disease: pre-symptomatic (P40), early symptomatic (P60), symptomatic (P90) and late symptomatic (P120).. Protein expression of interleukin (IL)-6 (26 kDa) was analyzed in spinal cord (A, $B), E D L(C, D)$ and SOL $(E, F)$. The housekeeping proteins selected to normalize the results were actin beta actin (ACTB) (42 kDa) and glyceraldehyde-3-phosphate dehydrogenase (GAPDH) (37 kDa). The sample size was 4 WT and 4 SOD1G93A mice. Unrelated t-test or Mann-Whitney U-tests were used. Graphics 
show relative mean \pm standard error of the mean. ${ }^{*} p<0.05$, ${ }^{\star *} p<0.01,{ }^{\wedge} p<0.10$. IL-6 $=$ interleukin $6, W T=$ wildtype, $\mathrm{EDL}=$ extensor digital longus muscle, $\mathrm{SOL}=$ soleus muscle, $\mathrm{ACTB}=$ actin beta, $\mathrm{GAPDH}=$ Glyceraldehyde-3-phosphate dehydrogenase

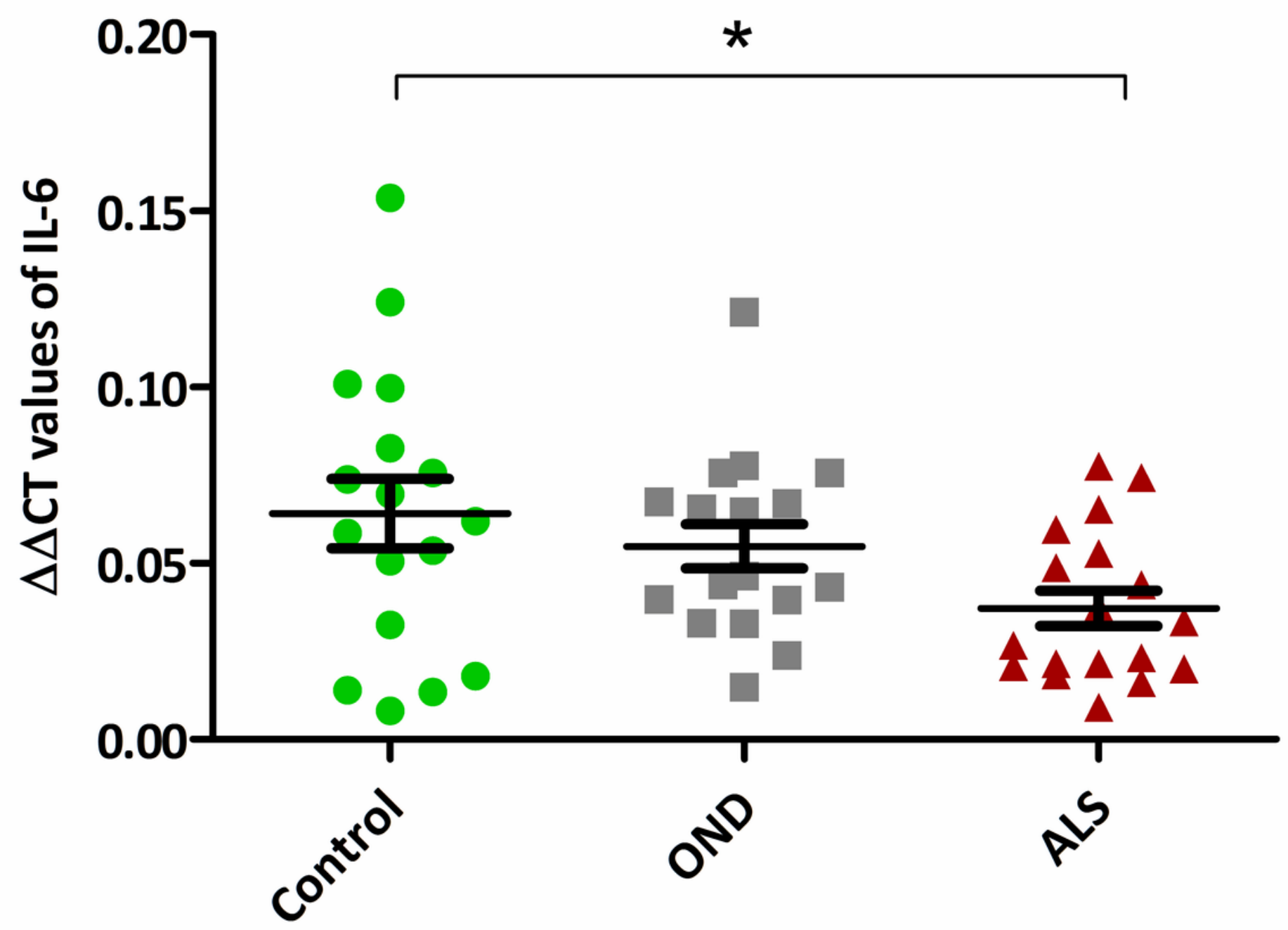

Figure 2

IL-6 gene expression was downregulated in blood from ALS patients. Gene expression of IL-6 was measured by qPCR in 18 ALS patients, 20 ONP patients and 20 control subjects. One-way ANOVA test and Bonferroni post-hoc were used. Graphic shows relative mean \pm standard error of the mean. ${ }^{*} p<0.05$. IL-6 = interleukin 6, ALS = amyotrophic lateral sclerosis, ONP = other neuropathies 


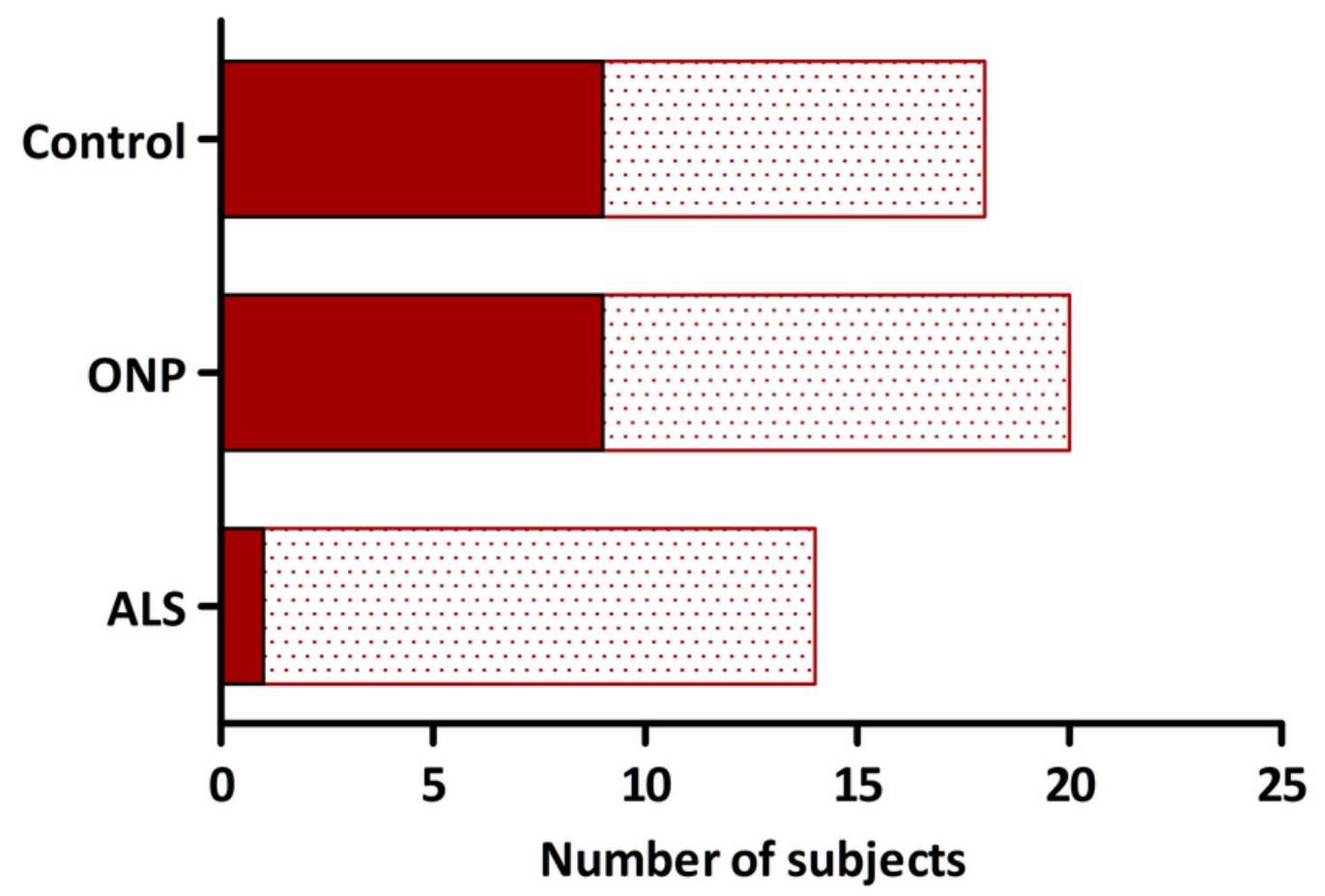

$\square$ Presence of IL-6

..... Absence of IL-6

Figure 3

Number of subjects showing presence or absence of IL-6 protein in plasma and grouped by the disease condition. The relation between the presence/absence of IL- 6 in blood of ALS patients $(n=14)$, ONP patients $(n=20)$ and controls $(n=18)$ was evaluated by a Chi-squared test $(A)$. The difference in proportions was found significant, $\chi^{2}(2, N=52)=7.241, p=0.027$. Graphic shows absolute number of subjects $(B) . I L-6=$ interleukin $6, A L S=$ amyotrophic lateral sclerosis, ONP = other neuropathies 